\title{
Pseudo-Linear Mechanics with Pseudo-Translation Symmetry
}

\author{
Wonsang Chung1*, Yeounju Kim¹, Seohyeon Kim¹, Jeongmin Kwon ${ }^{2}$ \\ ${ }^{1}$ Department of Physics and Research Institute of Natural Science, College of Natural Science, Gyeongsang National University, \\ Jinju, Korea \\ ${ }^{2}$ Department of Mathematics, College of Natural Science, Gyeongsang National University, Jinju, Korea \\ Email: *mimip44@naver.com
}

How to cite this paper: Chung, W., Kim, Y., Kim, S. and Kwon, J. (2021) Pseudo-Linear Mechanics with Pseudo-Translation Symmetry. Journal of Applied Mathematics and Physics, 9, 648-660.

https://doi.org/10.4236/jamp.2021.94047

Received: February 19, 2021

Accepted: April 18, 2021

Published: April 21, 2021

Copyright $\odot 2021$ by author(s) and Scientific Research Publishing Inc. This work is licensed under the Creative Commons Attribution International License (CC BY 4.0).

http://creativecommons.org/licenses/by/4.0/

\begin{abstract}
In this paper, we will consider the simplest pseudo-addition where pseudo-multiplication and pseudo-division are the same as the ordinary ones and distributivity hold. Using this we consider a special type of the non-uniform discrete times with the pseudo-translation symmetry. Besides, we demand that the displacement should be invariant under the pseudo-translation. From these, we obtain the new definition of the instantaneous velocity which is pseudo-translation symmetry in both time and position and formulate the mechanics which are pseudo-linear and pseudo-translation invariant.
\end{abstract}

\section{Keywords}

Pseudo-Multiplication, Pseudo-Addition, Pseudo-Translation Symmetry, Pseudo-Linear

\section{Introduction}

Newton equation in one dimension is given by

$$
M a=M \frac{\mathrm{d}}{\mathrm{d} t} v=M\left(\frac{\mathrm{d}}{\mathrm{d} t}\right)^{2} x=F,
$$

where $M$ denotes a particle mass. This gives the linear differential equation because $\frac{\mathrm{d}}{\mathrm{d} t}$ is linear. This derivative with respect to time comes from the Galilei's definition of the average velocity,

$$
\bar{v}=\frac{x\left(t^{\prime}\right)-x(t)}{t^{\prime}-t}
$$

Indeed, taking the limit $t^{\prime} \rightarrow t$ we get the Newton's instantaneous velocity from Equation (2). The definition (2) has the translational symmetry, 


$$
x \rightarrow x+\delta x, \quad t \rightarrow t+\delta t
$$

where the former is related to conservation of linear momentum while the latter is related to conservation of energy.

Recently, the relation between the average velocity and the instantaneous velocity was reexamined from the help of the discrete time. Use of the discrete time in physics has long history. First use was focused on the discrete calculus of variations [1] [2]. After this, the discrete Nöther theorem was found [3]. The possibility that time could be regarded as a discrete dynamical variable was examined through all phases of mechanics [4]. Some progress has been accomplished in this direction [5] [6] [7]. Principles to be used in the construction of discrete time classical mechanics were discussed in [7], where the authors introduced the concept of virtual path and constructed the system functions from classical Lagrangians and Cadzow's variational principle was applied to the action sum and Maeda-Noether and Logan invariants of the motion were obtained.

In the discrete mechanics, we introduce the discrete times

$$
t_{n}=n \tau, \quad n=0,1,2, \cdots,
$$

which obeys

$$
t_{n+1}-t_{n}=\tau
$$

In a discrete mechanic, the instantaneous velocity is not defined, instead the average one is well defined. We need two adjacent discrete times for the discrete velocity, which is defined as

$$
v\left(t_{n}\right)=\frac{x\left(t_{n+1}\right)-x\left(t_{n}\right)}{t_{n+1}-t_{n}}=\frac{x\left(t_{n}+\tau\right)-x\left(t_{n}\right)}{\tau}, n=0,1,2, \cdots,
$$

The discrete velocity is invariant under the transform $t_{n} \rightarrow t_{n}+L \tau, L \in \mathbb{Z}$, which is the discrete version of time translation symmetry. When we consider continuum limit from discrete time mechanics, we should fix the discrete time corresponding to finite continuous time $t$. Let such a discrete time be $t_{N}$. Then, the instantaneous velocity $v(t)$ is obtained by taking limit $\tau \rightarrow 0$ or $N \rightarrow \infty$ with $N \tau=t$ fixed; indeed, we have

$$
\begin{aligned}
v(t) & =\lim _{N \rightarrow \infty, \tau \rightarrow 0, N \tau=t} v\left(t_{N}\right) \\
& =\lim _{\tau \rightarrow 0} \frac{x\left(t_{N+1}\right)-x\left(t_{N}\right)}{\tau} \\
& =\lim _{\tau \rightarrow 0} \frac{x(t+\tau)-x(t)}{\tau} \\
& =\dot{x}
\end{aligned}
$$

The velocity (7) does not depend on choice of origin, which comes from the translation invariance of velocity. Indeed, the velocity remains unaltered under $x(t) \rightarrow x(t)+\xi$, which is the translation symmetry in space. The discrete velocity can be written as

$$
v\left(t_{n}\right)=\Delta_{t} x\left(t_{n}\right)
$$

where the difference operator is 


$$
\Delta_{t} F(t)=\frac{F(t+\tau)-F(t)}{\tau}
$$

In the continuum limit, we have $\Delta_{t} \rightarrow \frac{\mathrm{d}}{\mathrm{d} t}$. The difference operator is linear,

$$
\Delta_{t}(a F(t)+b G(t))=a \Delta_{t} F(t)+b \Delta_{t} G(t)
$$

The discrete velocity (8) is invariant under the translation in time and space and the difference operator is linear because we used the ordinary addition rule.

Similarly, the discrete acceleration is defined from three adjacent positions as

$$
a\left(t_{n}\right)=\frac{x\left(t_{n+2}\right)-x\left(t_{n+1}\right)-\left(x\left(t_{n+1}\right)-x\left(t_{n}\right)\right)}{\tau^{2}}, n=0,1,2, \cdots
$$

which is invariant under the transform $t_{n} \rightarrow t_{n}+L \tau, L \in \mathbb{Z}$ and $x(t) \rightarrow x(t)+\xi$. In the continuum limit, we have

$$
\begin{aligned}
a(t) & =\lim _{N \rightarrow \infty, \tau \rightarrow 0, N \tau=t} a\left(t_{N}\right) \\
& =\lim _{\tau \rightarrow 0} \frac{x\left(t_{N+2}\right)-2 x\left(t_{N+1}\right)+x\left(t_{N}\right)}{\tau^{2}} \\
& =\lim _{\tau \rightarrow 0} \frac{x(t+2 \tau)-2 x(t+\tau)+x(t)}{\tau^{2}} \\
& =\ddot{x}
\end{aligned}
$$

What will happen if we adopt deformed addition rule instead of the ordinary addition rule? The general deformed addition was introduced in the pseudo-analysis [8]. Pseudo-analysis first appeared by a mathematician Pap [8], where pseudo-addition, pseudo-subtraction, pseudo-multiplication and pseudo-division were defined by

$$
\begin{aligned}
& x \oplus_{g} y=g^{-1}(g(x)+g(y)) \\
& x \odot_{g} y=g^{-1}(g(x)-g(y)) \\
& x \odot_{g} y=g^{-1}(g(x) g(y)) \\
& x \oslash_{g} y=g^{-1}(g(x) / g(y))
\end{aligned}
$$

where $g$-map is bijective and monotonous with $g(0)=0, g(1)=1$. Then, the pseudo-additive identity is 0 and pseudo-multiplicative identity is 1 . There can be many possible $g$ s. In this paper, we demand a constraint for $g$ so that it may obey

$$
g(x) g(y)=g(x y),
$$

which is the homomorphism for the ordinary multiplication. Then, the pseudo-multiplication reduces to the ordinary multiplication. Inserting $y=1 / x$ in Equation (14) we have

$$
g\left(\frac{1}{x}\right)=\frac{1}{g(x)}
$$

which renders the pseudo-division to be ordinary division. In this case the pseudo-addition is distributive,

$$
k\left(x \oplus_{g} y\right)=(k x) \oplus_{g}(k y), \quad k \in \mathbb{R}
$$


The general solution obeying Equation (15) and Equation (16) is

$$
g(x)=|x|^{\alpha-1} x, \quad \alpha \in \mathbb{R}
$$

Then, pseudo-addition and pseudo-subtraction are given by

$$
\begin{aligned}
& a \oplus b=\left.|a| a\right|^{\alpha-1}+\left.b|b|^{\alpha-1}\right|^{1 / \alpha-1}\left(a|a|^{\alpha-1}+b|b|^{\alpha-1}\right) \\
& a \ominus b=\left.|a| a\right|^{\alpha-1}-\left.b|b|^{\alpha-1}\right|^{1 / \alpha-1}\left(a|a|^{\alpha-1}-b|b|^{\alpha-1}\right)
\end{aligned}
$$

From now on we call these kinds of pseudo-addition and pseudo-subtraction the $\alpha$-addition and $\alpha$-subtraction.

In this paper, we use the pseudo-addition to consider the non-uniform discrete times with the pseudo-translation symmetry. Besides, we demand that the displacement should be invariant under the pseudo-translation. From these, we formulate the mechanics which are pseudo-linear and pseudo-translation invariant. This paper is organized as follows: In Section 2, we discuss the $\alpha$-discrete time and $\alpha$-linear discrete mechanics. In Section 3, we discuss the $\alpha$-Work and conservation of $\alpha$-energy. In Section 4 , we discuss the $\alpha$-harmonic oscillator.

\section{2. $\alpha$-Discrete Time and $\alpha$-Linear Discrete Mechanics}

In this paper, we are to consider a special type of the non-uniform discrete times which obey the following relation

$$
t_{n+1} \ominus t_{n}=\tau, \quad n=0,1,2, \cdots,
$$

which gives

$$
t_{n}=[n]_{\alpha} \tau
$$

where $\alpha$-number is defined as

$$
[z]_{\alpha}=|z|^{\frac{1}{\alpha}-1} z, \quad z \in \mathbb{C}
$$

From now on we will say that discrete times are $\alpha$-uniform when they obey Equation (20). Besides, we refer to $\tau$ as the $\alpha$-uniform time interval. We also refer to the $\alpha$-uniform discrete times as shortly $\alpha$-discrete times. We stress that $\alpha$-uniform is not uniform unless $\alpha=1$. The $\alpha$-discrete times become the uniform discrete times when $\alpha=1$ is chosen. Thus, $|\alpha-1|$ denotes the degree of broken uniformity in discrete times.

\section{1. $\alpha$-Discrete Velocity}

In a uniform time lattice the discrete velocity is written as

$$
v\left(t_{n}\right)=\frac{\text { displacement }}{\text { uniform time interval }},
$$

where the uniform time interval is invariant under the translation $t_{n} \rightarrow t_{n}+L \tau$ and displacement is invariant under the translation $x \rightarrow x+\delta x$.

Now we will define the $\alpha$-discrete velocity as 


$$
v\left(t_{n}\right)=\frac{\alpha \text {-displacement }}{\alpha \text {-uniform time interval }}
$$

Here we know that $\alpha$-uniform time interval is invariant under the discrete $\alpha$-translation, $t_{n} \rightarrow t_{n} \oplus[L]_{\alpha} \tau$. Similarly, we define the $\alpha$-displacement so that it may be invariant under the continuous $\alpha$-translation $x \rightarrow x \oplus \delta x$. For two adjacent $\alpha$-discrete times, the $\alpha$-displacement is given by

$$
\alpha \text {-displacement }=x\left(t_{n+1}\right) \ominus x\left(t_{n}\right)
$$

Then, the $\alpha$-discrete velocity is defined as

$$
v_{\alpha}\left(t_{n}\right)=\frac{x\left(t_{n+1}\right) \ominus x\left(t_{n}\right)}{t_{n+1} \ominus t_{n}}=\frac{x\left(t_{n} \oplus \tau\right) \ominus x\left(t_{n}\right)}{\tau}, n=0,1,2, \cdots,
$$

The $\alpha$-discrete velocity is invariant under the discrete $\alpha$-translation. The $\alpha$-discrete velocity can also be written as

$$
v_{\alpha}\left(t_{n}\right)=\Delta_{t}^{\alpha} x\left(t_{n}\right),
$$

where the $\alpha$-difference operator is defined as

$$
\Delta_{t}^{\alpha} F(t)=\frac{F(t \oplus \tau) \ominus F(t)}{\tau}
$$

The $\alpha$-difference operator is not linear but obeys the $\alpha$-linearity,

$$
\Delta_{t}^{\alpha}(a F(t) \oplus b G(t))=a \Delta_{t}^{\alpha} F(t) \oplus b \Delta_{t}^{\alpha} G(t)
$$

The $\alpha$-discrete velocity remains unaltered under $x(t) \rightarrow x(t) \oplus \delta x(t)$, which is the $\alpha$-translation symmetry in space.

\section{2. $\alpha$-Velocity}

The continuum limit is obtained by taking the limit $\tau \rightarrow 0$ or $N \rightarrow \infty$ with $[N]_{\alpha} \tau=t$ fixed. Due to the $\alpha$-uniformity in time, the $\alpha$-discrete velocity does not give the ordinary Newton velocity in the continuum limit. Indeed we have

$$
\begin{aligned}
v_{\alpha}(t) & =\lim _{N \rightarrow \infty, \tau \rightarrow 0,[N]_{\alpha} \tau=t} v_{\alpha}\left(t_{N}\right) \\
& =\lim _{\tau \rightarrow 0} \frac{x\left(t_{N+1}\right) \ominus x\left(t_{N}\right)}{\tau} \\
& =\lim _{\tau \rightarrow 0} \frac{x(t \oplus \tau) \ominus x(t)}{\tau} \\
& =D_{t}^{\alpha} x(t),
\end{aligned}
$$

where the $\alpha$-derivative is defined as

$$
D_{t}^{\alpha} x(t)=|t|^{\frac{1}{\alpha}-1}|x(t)|^{1-\frac{1}{\alpha}}\left|\frac{\mathrm{d} x}{\mathrm{~d} t}\right|^{\frac{1}{\alpha}-1} \frac{\mathrm{d} x}{\mathrm{~d} t}
$$

The derivation of Equation (31) is given in Appendix A. The $\alpha$-derivative is $\alpha$-linear in the sense that

$$
D_{t}^{\alpha}\left(c_{1} x(t) \oplus c_{2} y(t)\right)=c_{1} D_{t}^{\alpha} x(t) \oplus c_{2} D_{t}^{\alpha} y(t)
$$

For the $\alpha$-derivative, the following Leibniz rule holds: 


$$
D_{t}^{\alpha}[F(t) G(t)]=\left(D_{t}^{\alpha} F(t)\right) G(t) \oplus F(t)\left(D_{t}^{\alpha} G(t)\right)
$$

We know that the $\alpha$-velocity (30) remains invariant under $\alpha$-translation in both time and space.

\section{3. $\alpha$-Discrete Acceleration and $\alpha$-Discrete Newton Equation}

The $\alpha$-discrete acceleration is obtained like Equation (12) in the form

$$
\begin{aligned}
a_{\alpha}\left(t_{n}\right) & =\frac{\left(x\left(t_{n+2}\right) \ominus x\left(t_{n+1}\right)\right) \ominus\left(x\left(t_{n+1}\right) \ominus x\left(t_{n}\right)\right)}{\left(t_{n+1} \ominus t_{n}\right)^{2}} \\
& =\frac{x\left(t_{n+2}\right) \ominus[2]_{\alpha} x\left(t_{n+1}\right) \oplus x\left(t_{n}\right)}{\tau^{2}}, n=0,1,2, \cdots,
\end{aligned}
$$

The $\alpha$-discrete acceleration can also be written as

$$
a_{\alpha}\left(t_{n}\right)=\left(\Delta_{t}^{\alpha}\right)^{2} x\left(t_{n}\right)=\Delta_{t}^{\alpha} v_{\alpha}\left(t_{n}\right)
$$

The $\alpha$-discrete acceleration remains unaltered under the transform $t_{n} \rightarrow t_{n} \oplus[L]_{\alpha} \tau, L \in \mathbb{Z}$ and $x(t) \rightarrow x(t) \oplus \delta x(t)$. The $\alpha$-discrete Newton equation is then given by

$$
M a_{\alpha}\left(t_{n}\right)=M D_{t}^{\alpha} v_{\alpha}\left(t_{n}\right)=M\left(D_{t}^{\alpha}\right)^{2} x\left(t_{n}\right)=F\left(t_{n}, x\left(t_{n}\right)\right)
$$

\section{4. $\alpha$-Acceleration and $\alpha$-Newton Equation}

In the continuum limit, $\alpha$-discrete acceleration reduces to the $\alpha$-acceleration

$$
a_{\alpha}(t)=D_{t}^{\alpha} v_{\alpha}(t)=\left(D_{t}^{\alpha}\right)^{2} x(t)
$$

which is invariant under $\alpha$-translation in both time and space. The Newton equation with $\alpha$-translation symmetry (or shortly $\alpha$-Newton equation) is then given by

$$
M a_{\alpha}(t)=M D_{t}^{\alpha} v_{\alpha}(t)=M\left(D_{t}^{\alpha}\right)^{2} x(t)=F
$$

Because $D_{t}^{\alpha}$ is $\alpha$-linear, we define the $\alpha$-net force for forces, $F_{1}, F_{2}, \cdots$, acted on the particle as

$$
F_{\alpha, \text { net }}=F_{1} \oplus F_{2} \oplus \cdots
$$

\section{3. $\alpha$-Work and Conservation of $\alpha$-Energy}

Now let us introduce the $\alpha$-work by the constant force $F$ in the following definition,

$$
W_{\alpha}=(\text { Force }) \times(\alpha \text {-displacement })
$$

If we consider that the particle moves from $x_{1}$ to $x_{2}$, we can write the $\alpha$-work by the constant force $F$,

$$
W_{\alpha}^{1 \rightarrow 2}=F\left(x_{2} \ominus x_{1}\right)
$$

Now let us consider the case that the force varies with the position and the 
particle moves from $x_{0}$ to $x_{N}$ in the discrete positions $\left\{x_{0}, x_{1}, \cdots, x_{N}\right\}$. Let $\bar{F}_{i, i+1}$ be the $\alpha$-average force when the particle moves from $x_{i}$ to $x_{i+1}$. Then, the $\alpha$-work is given by

$$
W_{\alpha}^{i \rightarrow i+1}=\bar{F}_{i, i+1}\left(x_{i+1} \ominus x_{i}\right)
$$

Then, the $\alpha$-work from $x_{0}$ to $x_{N}$ is

$$
W_{\alpha}^{0 \rightarrow N}=\bigoplus_{i=0}^{N-1} \bar{F}_{i, i+1}\left(x_{i+1} \ominus x_{i}\right),
$$

where $\alpha$-sum is define as

$$
\bigoplus_{i=0}^{N-1} A_{i}=A_{0} \oplus A_{1} \oplus \cdots \oplus A_{N-1}
$$

The $\alpha$-sum can be written in terms of the ordinary sum as

$$
\bigoplus_{i=0}^{N-1} A_{i}=\left.\left.\left|\sum_{i=0}^{N-1}\right| A_{i}\right|^{\alpha-1} A_{i}\right|^{\frac{1}{\alpha}-1} \sum_{i=0}^{N-1}\left|A_{i}\right|^{\alpha-1} A_{i}
$$

Now let us the continuum version of Equation (45) which is the $\alpha$-integral. The $\alpha$-integral is defined as

$$
\int_{a}^{x} \mathrm{~d}_{\alpha} \xi F(\xi)=\left.\left.\left|\int_{a}^{x} \alpha \mathrm{d} \xi\right| \xi\right|^{\alpha-1}|F(\xi)|^{\alpha-1} F(\xi)\right|^{\frac{1}{\alpha}-1} \int_{a}^{x} \alpha \mathrm{d} \xi|\xi|^{\alpha-1}|F(\xi)|^{\alpha-1} F(\xi)
$$

Here we know

$$
\int_{a}^{x} \mathrm{~d}_{\alpha} \xi\left(D_{\xi}^{\alpha} F(\xi)\right)=F(x) \ominus F(0)
$$

and

$$
D_{x}^{\alpha} \int_{a}^{x} \mathrm{~d}_{\alpha} \xi F(\xi)=F(x)
$$

The derivation of Equation (47) and Equation (48) is given in Appendix B.

With a help of the $\alpha$-integral, we define the $\alpha$-work from the position 1 to 2 for the varying force $F(x)$ as

$$
W_{\alpha}^{1 \rightarrow 2}=\int_{1}^{2} d_{\alpha} x F(x)
$$

Using the $\alpha$-Newton equation, we have

$$
W=K_{\alpha, 2} \odot K_{\alpha, 1}
$$
where $K_{\alpha}=\frac{1}{[2]_{\alpha}} M v^{2}$ is a $\alpha$-kinetic energy. The derivation is given in Appen-
$\operatorname{dix~C.~}$

If we introduce the $\alpha$-potential energy $U_{\alpha}(x)$ as

$$
F=-D_{x}^{\alpha} U_{\alpha}(x)
$$

and inserting it into the definition of the $\alpha$-work, we have

$$
W=-\left(U_{\alpha, 2} \ominus U_{\alpha, 1}\right)
$$

Thus, the conservation of $\alpha$-mechanical energy reads

$$
E_{\alpha}=K_{\alpha} \oplus U_{\alpha}=\text { const }
$$




\section{4. $\alpha$-Harmonic Oscillator}

As an example, we discuss the $\alpha$-harmonic oscillator problem. In the $\alpha$-discrete time mechanics, the harmonic oscillator is described by

$$
M\left(\frac{x\left(t_{n+2}\right) \ominus[2]_{\alpha} x\left(t_{n+1}\right) \oplus x\left(t_{n}\right)}{\tau^{2}}\right)=-M w^{2} x\left(t_{n}\right), \quad n=0,1,2, \cdots,
$$

or

$$
x\left(t_{n+2}\right) \ominus[2]_{\alpha} x\left(t_{n+1}\right) \oplus\left(1 \oplus \tau^{2} w^{2}\right) x\left(t_{n}\right)=0
$$

The initial condition is

$$
x\left(t_{0}\right)=A, \quad v_{\alpha}\left(t_{0}\right)=0
$$

Equation (55) is written as

$$
x\left(t_{n+2}\right) \ominus p x\left(t_{n+1}\right)=q\left(x\left(t_{n+1}\right) \ominus p x\left(t_{n}\right)\right),
$$

where

$$
p \oplus q=[2]_{\alpha}, \quad p q=1 \oplus w^{2} \tau^{2}
$$

Thus, we get

$$
\begin{aligned}
& p=1 \oplus i w \tau \\
& q=1 \ominus i w \tau,
\end{aligned}
$$

From the relation

$$
\begin{aligned}
x \oplus i y & =\left.|| x\right|^{\alpha-1} x+\left.|i y|^{\alpha-1} i y\right|^{\frac{1}{\alpha}-1}\left(|x|^{\alpha-1} x+|i y|^{\alpha-1} i y\right) \\
& =\left.|| x\right|^{\alpha-1} x+\left.i|y|^{\alpha-1} y\right|^{\frac{1}{\alpha}-1}\left(|x|^{\alpha-1} x+i|y|^{\alpha-1} y\right) \\
& =\left(\sqrt{|x|^{2 \alpha}+|y|^{2 \alpha}}\right)^{\frac{1}{\alpha}-1}\left(|x|^{\alpha-1} x+i|y|^{\alpha-1} y\right),
\end{aligned}
$$

we have

$$
\begin{aligned}
& p=\left[1+(w \tau)^{2 \alpha}\right]^{\frac{1-\alpha}{2 \alpha}}\left(1+i(w \tau)^{\alpha}\right) \\
& q=\left[1+(w \tau)^{2 \alpha}\right]^{\frac{1-\alpha}{2 \alpha}}\left(1-i(w \tau)^{\alpha}\right)
\end{aligned}
$$

Solving Equation (57) with initial condition, we get

$$
x\left(t_{n}\right)=\frac{A}{p \ominus q}\left[\left(p^{n} \ominus q^{n}\right) \ominus p q\left(p^{n-1} \ominus q^{n-1}\right)\right]
$$

One can set

$$
p=R \mathrm{e}^{i \theta}, q=R \mathrm{e}^{-i \theta},
$$

where

$$
R=\left(1+(w \tau)^{2 \alpha}\right)^{\frac{1}{2 \alpha}}
$$


and

$$
\theta=\tan ^{-1}(w \tau)^{\alpha}
$$

Then we get

$$
\begin{gathered}
p^{n} \ominus q^{n}=i[2]_{\alpha} R^{n}|\sin n \theta|^{\frac{1}{\alpha}-1} \sin n \theta \\
p q=R^{2}
\end{gathered}
$$

Thus, we have

$$
x\left(t_{n}\right)=A R^{n}|\cos n \theta|^{\frac{1}{\alpha}-1} \cos n \theta,
$$

The derivation of Equation (69) is given in Appendix D. In the continuum limit we have

$$
x(t)=A\left|\cos \left(w^{\alpha} t^{\alpha}\right)\right|^{\frac{1}{\alpha}-1} \cos \left(w^{\alpha} t^{\alpha}\right)
$$

Equation (70) is not periodic but it is $\alpha$-periodic in the sense,

$$
x\left(t \oplus T_{\alpha}\right)=x(t),
$$

where we call $T_{\alpha}$ the $\alpha$-period and it is given by

$$
T_{\alpha}=\frac{[2 \pi]_{\alpha}}{w}
$$

\section{Conclusion}

The uniform discrete times are related to the definition of velocity proposed by Newton. This is given by the ordinary derivative of the position with respect to time. In this paper, we considered a special type of the non-uniform discrete times which are related to pseudo calculus. Here we considered the special pseudo calculus called $\alpha$-calculus where $\alpha$-multiplication and $\alpha$-division are the same as the ordinary ones and distributivity holds. We considered the time pseudo lattice with the pseudo-translation symmetry described by the $\alpha$-addition. Besides, we demand that the displacement should be invariant under the pseudo-translation. From these, we obtained the new definition of the instantaneous velocity called $\alpha$-velocity which is pseudo-translation symmetry in both time and position. With the $\alpha$-velocity we formulated the mechanics which are pseudo-linear and pseudo-translation invariant. As an example, we discussed the simple harmonic oscillator in the $\alpha$-mechanics.

\section{Conflicts of Interest}

The authors declare no conflicts of interest regarding the publication of this paper.

\section{References}

[1] Cadzow, J.A. (1970) Discrete Calculus of Variations. International Journal of Control, 11, 393-407. https://doi.org/10.1080/00207177008905922 
[2] Logan, J.D. (1973) First Integrals in the Discrete Variational Calculus. Aequationes Mathematicae, 9, 210-220. https://doi.org/10.1007/BF01832628

[3] Maeda, S. (1981) Extension of Discrete Noether Theorem. Mathematica Japonica, 26, 85-90.

[4] Lee, T.D. (1983) Can Time Be Discrete Dynamical Variable? Physics Letters B, 122, 217-220. https://doi.org/10.1016/0370-2693(83)90687-1

[5] Yamamoto, H., Hayashi, A., Hashimoto, T. and Horibe, M. (1995) Conserved Quantities of Field Theory on Discrete Space-Time. Progress of Theoretical Physics, 93, 173-184. https://doi.org/10.1143/ptp/93.1.173

[6] Hashimoto, T., Hayashi, A., Horibe, M. and Yamamoto, H. (1995) How to Quantize Fields Canonically on Discrete Space-Time. Progress of Theoretical Physics, 94, 249-261. https://doi.org/10.1143/PTP.94.249

[7] Jaroszkiewicz, G. and Norton, K. (1997) Principles of Discrete Time Mechanics: I. Particle Systems. Journal of Physics A: Mathematical and General, 30, 3115-3144. https://doi.org/10.1088/0305-4470/30/9/022

[8] Pap, E. (1993) g-Calculus. Novi Sad Journal of Mathematics, 23, 145-156. 


\section{Appendix A}

The $\alpha$-addition and $\alpha$-subtraction depend on sign of two numbers.

Case of $x>0, y>0$ :

$$
\begin{gathered}
x \oplus y=\left(x^{\alpha}+y^{\alpha}\right)^{1 / \alpha} \\
x \ominus y= \begin{cases}\left(x^{\alpha}-y^{\alpha}\right)^{1 / \alpha} & (x>y) \\
-\left(y^{\alpha}-x^{\alpha}\right)^{1 / \alpha} & (x<y)\end{cases}
\end{gathered}
$$

Case of $x>0, y<0$ :

$$
\begin{gathered}
x \oplus y= \begin{cases}\left(x^{\alpha}-(-y)^{\alpha}\right)^{1 / \alpha} & (x>-y) \\
-\left((-y)^{\alpha}-x^{\alpha}\right)^{1 / \alpha} & (x<-y)\end{cases} \\
x \ominus y=\left(x^{\alpha}+(-y)^{\alpha}\right)^{1 / \alpha}
\end{gathered}
$$

Case of $x<0, y>0$ :

$$
\begin{gathered}
x \oplus y= \begin{cases}\left(y^{\alpha}-(-x)^{\alpha}\right)^{1 / \alpha} & (-x<y) \\
-\left((-x)^{\alpha}-y^{\alpha}\right)^{1 / \alpha} & (-x>y)\end{cases} \\
x \in y=-\left(y^{\alpha}+(-x)^{\alpha}\right)^{1 / \alpha}
\end{gathered}
$$

Case of $x<0, y<0$ :

$$
\begin{gathered}
x \oplus y=-\left((-x)^{\alpha}+(-y)^{\alpha}\right)^{1 / \alpha} \\
x \oplus y= \begin{cases}\left((-y)^{\alpha}-(-x)^{\alpha}\right)^{1 / \alpha} & (x>y) \\
-\left((-x)^{\alpha}-(-y)^{\alpha}\right)^{1 / \alpha} & (x<y)\end{cases}
\end{gathered}
$$

For a small $\tau_{\alpha}$, we have

$$
t \Theta_{\alpha} \tau_{\alpha}=\left(t^{\alpha}-\tau_{\alpha}^{\alpha}\right)^{1 / \alpha}=t\left(1-\frac{\tau_{\alpha}^{\alpha}}{t^{\alpha}}\right)^{1 / \alpha} \approx t-\frac{1}{\alpha} t^{1-\alpha} \tau_{\alpha}^{\alpha}
$$

and

$$
x\left(t \Theta_{\alpha} \tau_{\alpha}\right) \approx x(t)-\frac{1}{\alpha} t^{1-\alpha} \tau_{\alpha}^{\alpha} \dot{x}(t)
$$

Now let us consider the following four cases.

Case of $x(t)>0, \dot{x}(t)>0:$ In this case we have

$$
x(t)>x\left(t \ominus_{\alpha} \tau_{\alpha}\right)>0
$$

Hence we get

$$
D_{t} x(t)=\lim _{\tau_{\alpha} \rightarrow 0} \frac{\left(x(t)^{\alpha}-\left(x(t)-\frac{1}{\alpha} t^{1-\alpha} \tau_{\alpha}^{\alpha} \dot{x}(t)\right)^{\alpha}\right)^{1 / \alpha}}{\tau_{\alpha}}=t^{\frac{1}{\alpha}-1} x(t)^{1-\frac{1}{\alpha}}(\dot{x})^{\frac{1}{\alpha}}
$$


Case of $x(t)>0, \dot{x}(t)<0$ : In this case we have

$$
x\left(t \ominus_{\alpha} \tau_{\alpha}\right)>x(t)>0
$$

Hence we get

$$
D_{t} x(t)=-\lim _{\tau_{\alpha} \rightarrow 0} \frac{\left(\left(x(t)-\frac{1}{\alpha} t^{1-\alpha} \tau_{\alpha}^{\alpha} \dot{x}(t)\right)^{\alpha}-x(t)^{\alpha}\right)^{1 / \alpha}}{\tau_{\alpha}}=-t^{\frac{1}{\alpha}-1} x(t)^{1-\frac{1}{\alpha}}|\dot{x}|^{\frac{1}{\alpha}}
$$

Case of $x(t)<0, \dot{x}(t)>0$ : In this case we have

$$
x\left(t \Theta_{\alpha} \tau_{\alpha}\right)<x(t)<0
$$

Hence we get

$$
D_{t} x(t)=\lim _{\tau_{\alpha} \rightarrow 0} \frac{\left(\left(|x(t)|+\frac{1}{\alpha} t^{1-\alpha} \tau_{\alpha}^{\alpha} \dot{x}(t)\right)^{\alpha}-|x(t)|^{\alpha}\right)^{1 / \alpha}}{\tau_{\alpha}}=t^{\frac{1}{\alpha}-1}|x(t)|^{1-\frac{1}{\alpha}}(\dot{x})^{\frac{1}{\alpha}}
$$

Case of $x(t)<0, \dot{x}(t)<0$ : In this case we have

$$
x(t)<x\left(t \Theta_{\alpha} \tau_{\alpha}\right)<0
$$

Hence we get

$$
D_{t} x(t)=-\lim _{\tau_{\alpha} \rightarrow 0} \frac{\left(|x(t)|^{\alpha}-\left(|x(t)|+\frac{1}{\alpha} t^{1-\alpha} \tau_{\alpha}^{\alpha} \dot{x}(t)\right)^{\alpha}\right)^{1 / \alpha}}{\tau_{\alpha}}=-t^{\frac{1}{\alpha}-1}|x(t)|^{1-\frac{1}{\alpha}}|\dot{x}|^{\frac{1}{\alpha}}
$$

\section{Appendix B}

We have

$$
\begin{aligned}
& \int_{a}^{x} \mathrm{~d}_{\alpha} \xi\left(D_{\xi}^{\alpha} F(\xi)\right) \\
& =\left.\left.\left|\int_{a}^{x} \alpha \mathrm{d} \xi\right| \xi\right|^{\alpha-1}\left|D_{\xi}^{\alpha} F(\xi)\right|^{\alpha-1} D_{\xi}^{\alpha} F(\xi)\right|^{\frac{1}{\alpha}-1} \int_{a}^{x} \alpha \mathrm{d} \xi|\xi|^{\alpha-1}\left|D_{\xi}^{\alpha} F(\xi)\right|^{\alpha-1} D_{\xi}^{\alpha} F(\xi) \\
& =\left.\left.\left|\int_{a}^{x} \alpha \mathrm{d} \xi\right| F(\xi)\right|^{\alpha-1} F^{\prime}(\xi)\right|^{\frac{1}{\alpha}-1} \int_{a}^{x} \alpha \mathrm{d} \xi|F(\xi)|^{\alpha-1} F^{\prime}(\xi) \\
& =\left.|| F(x)\right|^{\alpha-1} F(x)-\left.|F(a)|^{\alpha-1} F(a)\right|^{\frac{1}{\alpha}-1}\left(|F(x)|^{\alpha-1} F(x)-|F(a)|^{\alpha-1} F(a)\right) \\
& =F(x) \ominus F(a)
\end{aligned}
$$

where we used

$$
\left|D_{\xi}^{\alpha} F(\xi)\right|^{\alpha-1} D_{\xi}^{\alpha} F(\xi)=|\xi|^{1-\alpha}|F(\xi)|^{\alpha-1} F^{\prime}(\xi)
$$

\section{Appendix C}

From the definition of $\alpha$-work, we have

$$
\begin{aligned}
W_{\alpha}^{1 \rightarrow 2} & =\int_{1}^{2} \mathrm{~d}_{\alpha} x F(x) \\
& =\left.\left.\left|\int_{1}^{2} \alpha \mathrm{d} x\right| x\right|^{\alpha-1}|F(x)|^{\alpha-1} F(x)\right|^{\frac{1}{\alpha}-1} \int_{1}^{2} \alpha \mathrm{d} x|x|^{\alpha-1}|F(x)|^{\alpha-1} F(x)
\end{aligned}
$$


Now let us set

$$
A=\int_{1}^{2} \alpha \mathrm{d} x|x|^{\alpha-1}|F(x)|^{\alpha-1} F(x)
$$

Using $\alpha$-Newton equation, we have

$$
\begin{aligned}
A & =M^{\alpha} \int_{1}^{2} \alpha \mathrm{d} t \dot{x}|x|^{\alpha-1}\left|D_{t}^{\alpha} v\right|^{\alpha-1} D_{t}^{\alpha} v \\
& =M^{\alpha} \int_{1}^{2} \alpha \mathrm{d} t t^{1-\alpha} \dot{x}|x|^{\alpha-1}|v|^{\alpha-1} \dot{v} \\
& =M^{\alpha} \int_{1}^{2} \alpha \mathrm{d} t v|v|^{2 \alpha-2} \dot{v}
\end{aligned}
$$

Thus we have

$$
W_{\alpha}^{1 \rightarrow 2}=\left.\left.M\left|\int_{1}^{2} \alpha \mathrm{d} t v\right| v\right|^{2 \alpha-2} \dot{v}\right|^{\frac{1}{\alpha}-1} \int_{1}^{2} \alpha \mathrm{d} t v|v|^{2 \alpha-2} \dot{v}
$$

Also, we have

$$
\begin{aligned}
& \int_{1}^{2} \mathrm{~d}_{\alpha} t D_{t}^{\alpha}\left(\frac{1}{[2]_{\alpha}} M v^{2}\right)=M \int_{1}^{2} \mathrm{~d}_{\alpha} t v D_{t}^{\alpha} v \\
& =\left.\left.M\left|\int_{1}^{2} \alpha \mathrm{d} t t^{\alpha-1}\right| v\right|^{\alpha-1} v\left|D_{t}^{\alpha} v\right|^{\alpha-1} D_{t}^{\alpha} v\right|^{\frac{1}{\alpha}-1} \int_{1}^{2} \alpha \mathrm{d} t t^{\alpha-1}|v|^{\alpha-1} v\left|D_{t}^{\alpha} v\right|^{\alpha-1} D_{t}^{\alpha} v \\
& =\left.\left.M\left|\int_{1}^{2} \alpha \mathrm{d} t v\right| v\right|^{2 \alpha-2} \dot{v}\right|^{\frac{1}{\alpha}-1} \int_{1}^{2} \alpha \mathrm{d} t v|v|^{2 \alpha-2} \dot{v}
\end{aligned}
$$

\section{Appendix D}

Inserting Equation (67) and Equation (68) into Equation (63), we get

$$
x\left(t_{n}\right)=\frac{A R^{n}}{w \tau}\left(|\sin n \theta|^{\frac{1}{\alpha}-1} \sin n \theta \ominus R|\sin (n-1) \theta|^{\frac{1}{\alpha}-1} \sin (n-1) \theta\right)
$$

Here we have

$$
\begin{aligned}
& |\sin n \theta|^{\frac{1}{\alpha}-1} \sin n \theta \ominus R|\sin (n-1) \theta|^{\frac{1}{\alpha}-1} \sin (n-1) \theta \\
& =\left|\sin n \theta-R^{\alpha} \sin (n-1) \theta\right|^{\frac{1}{\alpha}-1}\left(\sin n \theta-R^{\alpha} \sin (n-1) \theta\right) \\
& =w \tau|\cos n \theta|^{\frac{1}{\alpha}-1} \cos n \theta,
\end{aligned}
$$

where we used

$$
R^{\alpha}=\sec \theta
$$

